\title{
EXPERIENCIAS CON UN GESTOR DE APLICACIONES BASADO EN LOCALIZACIÓN MEDIANTE REDES INALÁMBRICAS
}

\author{
(EXPERIENCES WITH AN APPLICATION MANAGER USING LOCATION BY WIRELESS LAN)
}

\author{
Sergio Martín \\ Manuel Castro \\ Rosario Gil \\ Juan Peire
}

Departamento de Ingeniería Electrónica, Eléctrica y de Control (DIEEC)

Escuela Técnica Superior de Ingenieros Industriales (ETSII) UNED. (España)

\section{RESUMEN}

La localización geográfica mediante redes inalámbricas puede mejorar la manera en que las Universidades se relacionan con sus alumnos y profesores, personalizando sus relaciones con ellos. Así, es posible, ofrecer contenidos en función de la localización geográfica y el perfil personal. Así mismo, esta tecnología puede ser aplicada con éxito a otros entornos como puede ser el turístico, mostrando la información de cada uno de los cuadros de un museo con solo acercarse al mismo; el sanitario, mostrando al personal sanitario información de un paciente con sólo entrar en su habitación; comercial, ofreciendo información de un stand comercial de un recinto ferial; industrial, etc.

\begin{abstract}
The geographic location by wireless LAN can improve the way in that the Universities relate with their students and professors, personalizing the relations with them. In that way, it is possible to offer contents in function of the geographic location of the user and his personal profile. In addition, this technology can be applied with success to others environments, as tourist, showing the information of every art work of a museum just by to approach; the hospital environment, offering the information of a patient with just enter to his room; marketing, showing information about a commercial stand in a congress area; industrial, etc.
\end{abstract}




\section{INTRODUCCIÓN}

En la actualidad la mayoría de las Universidades han implantado redes inalámbricas que les permiten ofrecer acceso a la Web a sus alumnos y personal universitario desde cualquier lugar del campus universitario. Los servicios desarrollados dentro del proyecto "Sistema de Gestión de Aplicaciones basado en localización mediante redes wlan" permiten a estas Universidades personalizar la información ofrecida en función de la localización del usuario en el recinto universitario. De esta manera, cuando por ejemplo un alumno entre en un laboratorio podrá obtener de forma automática información sobre la práctica que se esté llevando a cabo en él; de igual manera podrá obtener información administrativa con sólo acercarse a la secretaria del centro docente o acceder a la presentación que se está llevando a cabo en una sala de conferencias.

Aunque el proyecto ha sido concebido y desarrollado en este entorno particular de la enseñanza universitaria, los desarrollos llevados a cabo tienen un gran potencial de explotación comercial al ser el rango de posibles aplicaciones muy elevado. Basta con pensar las aplicaciones del sistema en hospitales (un médico puede obtener el historial de un paciente en su PDA con sólo acercarse a la habitación en la que se encuentra), museos (los visitantes podrían ir accediendo en tiempo real a la información sobre las diferentes salas por la que pasean o incluso sobre una cierta obra de arte con sólo situarse frente a ella), seguridad en edificios (los vigilantes pueden conocer en todo momento donde se encuentran los usuarios que lleven dispositivos inalámbricos y que ruta han seguido), cascos urbanos (los ciudadanos podrán obtener información turística o administrativa según se encuentren en una dependencia particular del ayuntamiento o frente a un museo o una iglesia), centros de congresos, etc.

\section{OBJETIVOS}

El objetivo principal del proyecto es estudiar las aplicaciones que, en el entorno universitario, tienen los sistemas de localización geográfica ofrecidos por la tecnología inalámbrica Wireless LAN. Aunque la instalación de redes sin cable en las universidades podría considerarse una actividad innovadora en sí misma, la unión de estas redes con sistemas que permitan la localización de los terminales del personal de la universidad y los alumnos en el campus universitario ofrece nuevas posibilidades que deberán ser estudiadas y explotadas.

El sistema deberá, en primer lugar, proporcionar la infraestructura necesaria para dar acceso inalámbrico a la red. A continuación, deberá localizar al usuario 
dentro de las distintas áreas de servicio o tecnológicas que se definan en cada edificio (despachos, salas de conferencia, laboratorios, etc.) y, por último, deberá filtrar y proporcionar los servicios de información adecuados en función del perfil y del área en el que se encuentre el usuario.

De esta manera, un usuario, dotado con un ordenador portátil (o una agenda electrónica tipo PDA) recibirá directamente, al entrar en cualquiera de las instalaciones, la información pertinente obtenida desde los servicios de información de la Universidad. Así, por ejemplo, un usuario conectado por red inalámbrica a la Universidad, recibiría automáticamente al entrar en una sala de conferencias la documentación relacionada (transparencias de la presentación, documentación adicional,CVdelponente, etc.). Deigualmanera, unalumnorecibiría automáticamente la información sobre una práctica de laboratorio con sólo acceder al lugar en el que éste se encuentra.

\section{DESCRIPCIÓN DE LA TECNOLOGÍA}

En el diseño de la herramienta ha tenido gran importancia la construcción de una arquitectura abierta y modular que permitiera la integración en diversos entornos adaptándose a los requerimientos del mercado en cada momento. Para lograr el reto de construir un sistema de tales características se ha diseñado el sistema de modo que presenta tres módulos principales: la red inalámbrica, el sistema de localización y el gestor de aplicaciones, tal y como se puede observar en la figura 1.

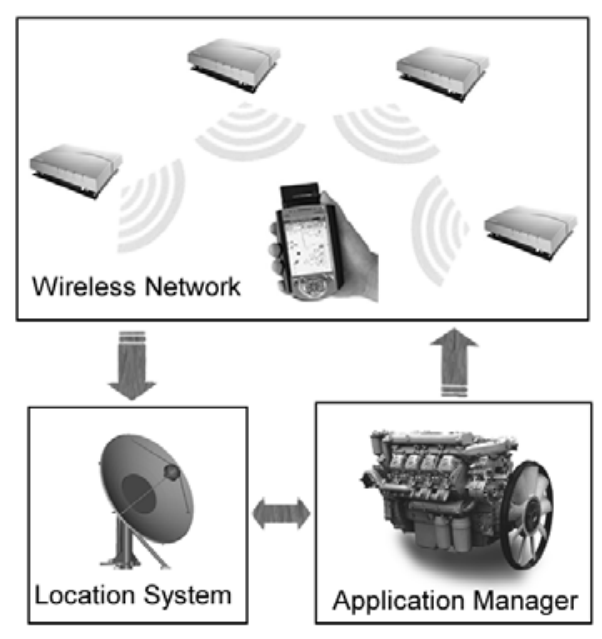

Fig. 1: Arquitectura lógica del sistema 


\section{Wireless LAN}

Aunque la red inalámbrica suele ser considerada más como un recurso empleado por el sistema que como un componente, en nuestro caso particular presenta ciertas características que afectan al desarrollo del prototipo. En particular, la implantación del producto final requiere de un estudio previo del entorno y de las condiciones que podrían afectar a los servicios de localización y que permita definir el número de puntos de acceso a instalar y determinar su distribución óptima.

La infraestructura de red inalámbrica empleada en el proyecto se basa en una serie de puntos de acceso que siguen el estándar 802.11b del IEEE (wi-fi) que ofrece un ancho de banda de hasta 11 Mbps y es, actualmente, el más ampliamente difundido en España (y en Europa).

Según esta norma, las tramas IP transmitidas entre los terminales y los puntos de acceso deben contener información sobre la potencia de la señal para permitir realizar el proceso de "asociación", es decir, elegir el punto de acceso al que debe conectarse el terminal en función de la potencia recibida y del ratio de error de paquetes que proporcione, siempre buscando los niveles óptimos. Así mismo, cuando dichos niveles se degradan o el cliente percibe que puede obtener mejores condiciones de señal y transmisión puede cambiar de punto de acceso. Es lo que se conoce como "reasociación", un proceso típico en los desplazamientos de los usuarios a través de las distintas coberturas de varios puntos de acceso. Este proceso dinámico de asociación y reasociación permite a los administradores de redes establecer WLAN con muy amplia cobertura creando una serie de células $11 \mathrm{~b}$ superpuestas a través de un edificio o área. Para ello, habrá que emplear reutilización de canal, evitando que los puntos de acceso sobre un canal DSSS se solapen con un canal utilizado por su punto de acceso vecino.

Además de la movilidad en la red que aportan estos dos procesos, ofrecen una ventaja añadida ya que es posible conocer en todo momento la potencia de la señal que recibe cada uno de los puntos de acceso que componen la red inalámbrica desde cada uno de los terminales conectados. Analizando la potencia de estas señales y realizando triangulaciones es posible determinar la situación relativa de un usuario respecto de los nodos de la red.

En un entorno ideal, estas triangulaciones deberían permitir localizar al emisor en su posición exacta. Sin embargo, la potencia que reciben los nodos de acceso de cada elemento de red no es constante y puede verse afectada por campos eléctricos (un 
microondas por ejemplo), físicos (tabiques de división de salas, despachos y zonas de paso que contienen materiales de aislamiento) o térmicos (afluencia de gente). Este hecho hace necesario medir la potencia y ruido de la señal entre los puntos de acceso de la red y las zonas de paso posibles con el fin de asegurar la máxima cobertura de red. Estas mediciones darán como resultado la colocación óptima de los puntos de acceso en la zona a cubrir por la red inalámbrica y servirán también para configurar y calibrar las redes neuronales que realizarán las triangulaciones de potencia.

Por ejemplo, en el prototipo instalado en las instalaciones del Departamento de Ingeniería Eléctrica, Electrónica y de Control de la Escuela Técnica Superior de Ingenieros Industriales de la UNED (Madrid) se ha definido una red local inalámbrica que cubre una planta de un área de $800 \mathrm{~m}^{2}$ aprox. con 5 puntos de acceso. Aunque para proporcionar cobertura wireless a toda la planta habría bastado dos puntos de acceso, para conseguir una localización más precisa es necesario tener al menos tres. Además, teniendo en cuenta las características de la planta se ha optado finalmente por instalar 5 puntos de acceso distribuidos tal y como se muestra en la figura 2.

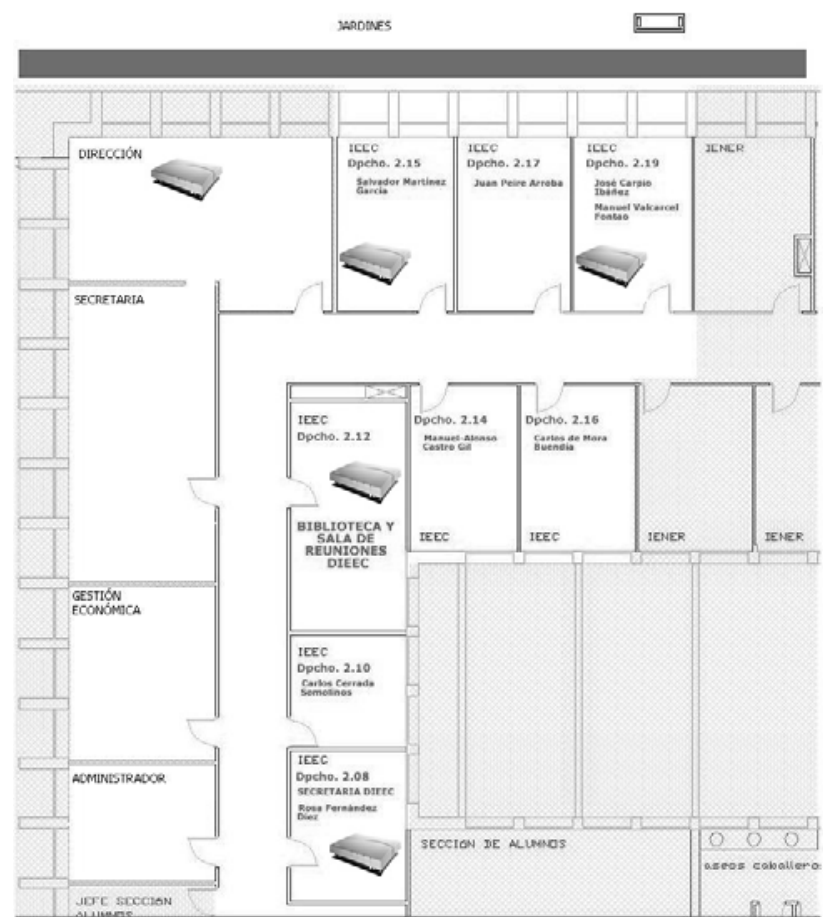

Figura 2: Mapa de distribución de puntos de acceso. 
Otro factor importante en el despliegue de cualquier red inalámbrica es su securización. Durante el proyecto se han empleado dos métodos. El primero es el cifrado de datos entre usuario y punto de acceso mediante WEP (Wired Equivalent Privacy, Privacidad Equivalente al Cable), el cual permite cifrar todas las conexiones de datos realizadas vía radio mediante un sistema criptográfico de clave simétrica. Este sistema no es muy seguro, ya que se ha demostrado que es posible obtener la clave simétrica de cifrado utilizando escuchas de las comunicaciones durante ciertos períodos de tiempo. El otro método utilizado para asegurar las comunicaciones fue la autenticación 802.1x con PEAP y MS CHAP v2. El método 802.1x permite realizar autenticación basada en el usuario, siendo posible la utilización de credenciales tales como contraseñas o certificados para asegurar la autenticidad del usuario, además posibilita el aprovechamiento de protocolos AAA tales como RADIUS para centralizar la autenticación y las autorizaciones, (figura 3). Las pruebas realizadas nos hicieron optar finalmente por el segundo sistema ya que la autentificación WEP no ofrece actualmente garantías suficientes en cuanto a seguridad. A continuación se muestra un esquema genérico de cómo funciona la autenticación de dispositivos wireless en nuestra red inalámbrica.

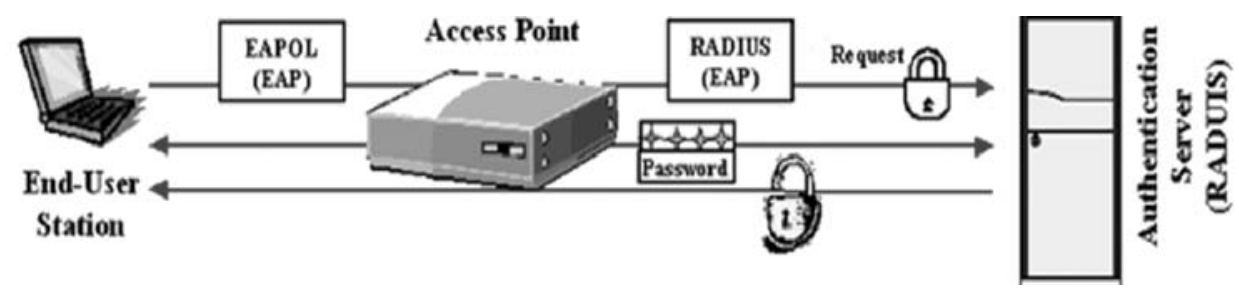

Figura 3: Esquema de seguridad del sistema: cifrado de datos y autenticación de usuarios.

Las redes inalámbricas han conseguido una gran popularidad en varios sectores tales como salud, logística, educación, distribución y producción. Estas sectores se han beneficiado de los aumentos de la productividad originados por la utilización de terminales de manos libres, "notebooks" y dispositivos portátiles para transmitir en tiempo real información a un servidor central para su posterior procesamiento. Hoy en día, las redes inalámbricas están siendo ampliamente consideradas por un gran número de sectores como una magnífica alternativa para sus necesidades de conectividad. En general, estas redes poseen una serie de características que las hacen muy atractivas, entre las que cabe destacar: 
- Movilidad: los sistemas inalámbricos facultan a los usuarios de redes de área local con acceso a información en tiempo real en cualquier lugar de la organización. Esta movilidad aumenta la productividad y las posibilidades de servicio que no eran posibles con redes cableadas.

- Velocidad de instalación y simplicidad: instalar un sistema inalámbrico puede ser muy rápido y fácil, aparte de eliminar la necesidad de cablear muros y techos.

- Flexibilidad: la tecnología inalámbrica puede alcanzar aquellos lugares cuya difícil accesibilidad desaconseja una solución cableada.

- Escalabilidad: las soluciones inalámbricas pueden ser configuradas en una enorme variedad de topologías para satisfacer las necesidades de instalaciones y aplicaciones específicas. Las configuraciones pueden cambiarse fácilmente y cubren desde pequeñas redes compuestas por unos pocos terminales, hasta soluciones más complejas conectando miles de ordenadores y dispositivos en un área determinada.

- Reducción del coste de propiedad: si bien la inversión inicial requerida por el hardware de una solución sin cable, puede ser mayor que el coste del hardware de una red cableada, los gastos generales de instalación y los costes a lo largo del ciclo de vida son significativamente menores. Los ahorros a largo plazo son mucho mayores en aquellos entornos dinámicos donde se producen frecuentes movimientos y cambios.

Al ser las redes inalámbricas $802.11 \mathrm{~B}$ una tecnología de plataforma independiente, puede funcionar en cualquier tipo de aplicaciones basadas en redes inalámbricas WLAN, como pudieran ser Bluetooth, 802.11A, HyperLAN2, y otro tipo de tecnologías de red. Es de fácil manejo e integrable y permite obtener la información de localización en tiempo real.

\section{Sistema de localización geográfica mediante wireless lan}

Uno de los servicios más innovadores para entornos WLAN es aquél que ofrece información de localización geográfica del terminal del usuario. Este servicio está basado en un motor de posicionamiento (Positioning Engine) capaz de localizar clientes inalámbricos, portátiles, agendas electrónicas y otros dispositivos que cumplan el estándar $802.11 \mathrm{~b}$, con una cierta precisión (los sistemas más avanzados 
tiene en la actualidad un margen de error de 1m, suficientemente pequeño para el tipo de aplicaciones que planteamos).

Básicamente, estos servicios ofrecen las coordenadas (X,Y,Z) de cada uno de los terminales inalámbricos conectados a la red. A partir de ellas, es posible determinar en qué zona o dependencia del edificio se encuentran dichos terminales. Con estos datos se determinará qué información debe ser ofrecida a cada terminal, así como realizar el seguimiento de los clientes inalámbricos.

Los sistemas más avanzados utilizan un algoritmo de cálculo de posiciones basado en unos modelos "cutting-edge" matemáticos y teorías de probabilidad, ofreciendo un resultado más robusto, con mayores prestaciones y proporcionando unas coordenadas más precisas que, por ejemplo, los algoritmos de triangulación u otros métodos de propagación. El sistema es además escalable, es decir, si se requiere una precisión mayor, bastará simplemente con añadir nuevos puntos de acceso a la red wi-fi.

Para obtener la información sobre la posición de un elemento de red a partir de las potencias emitidas es necesario obtener dichas potencias en tres o más puntos de acceso para poder determinar sus posiciones relativas mediante triangulación. Como ya hemos comentado las potencias recibidas no son constantes ya que pueden verse alteradas por la influencia de diferentes campos. Esta problemática se ha resuelto con la utilización de un software comercial de la casa Ekahau fruto de más de diez años de estudios de investigación realizados por un grupo especial de la Universidad de Helsinki (Finlandia).

Este sistema ofrece un conjunto de redes neuronales capaces de realizar las triangulaciones necesarias y ajustar los resultados, en cierta medida, a las condiciones medioambientales que se presenten. Estas redes neuronales requieren deuna calibración previa realizada siguiendo un patrón de posicionamiento en el que se definen los puntos geográficos más significativos de una dependencia física para los que se registran las potencias recibidas en todos los puntos de acceso instalados en la red desde un terminal móvil genérico.

Normalmente, estos servicios ofrecen un software al que se accede mediante un interfaz API JAVA e incorporan una aplicación de Calibración y una de Gestión que permite analizar la precisión de la localización, mostrando la cobertura de la red y los dispositivos inalámbricos rastreados.

El sistema requiere de una calibración previa para, primeramente crear un nuevo patrón de posicionamiento, (figura 4). Una vez creado, se calibra, moviéndose alrededor 
del área de localización y tomando muestras de referencias del nivel de señal en cada punto (SRI). Una vez calibrado podemos visualizar los vectores de error cuadrático medio de posicionamiento, áreas de cobertura, y niveles de señal para optimizar la red para la transferencia de datos y localización.

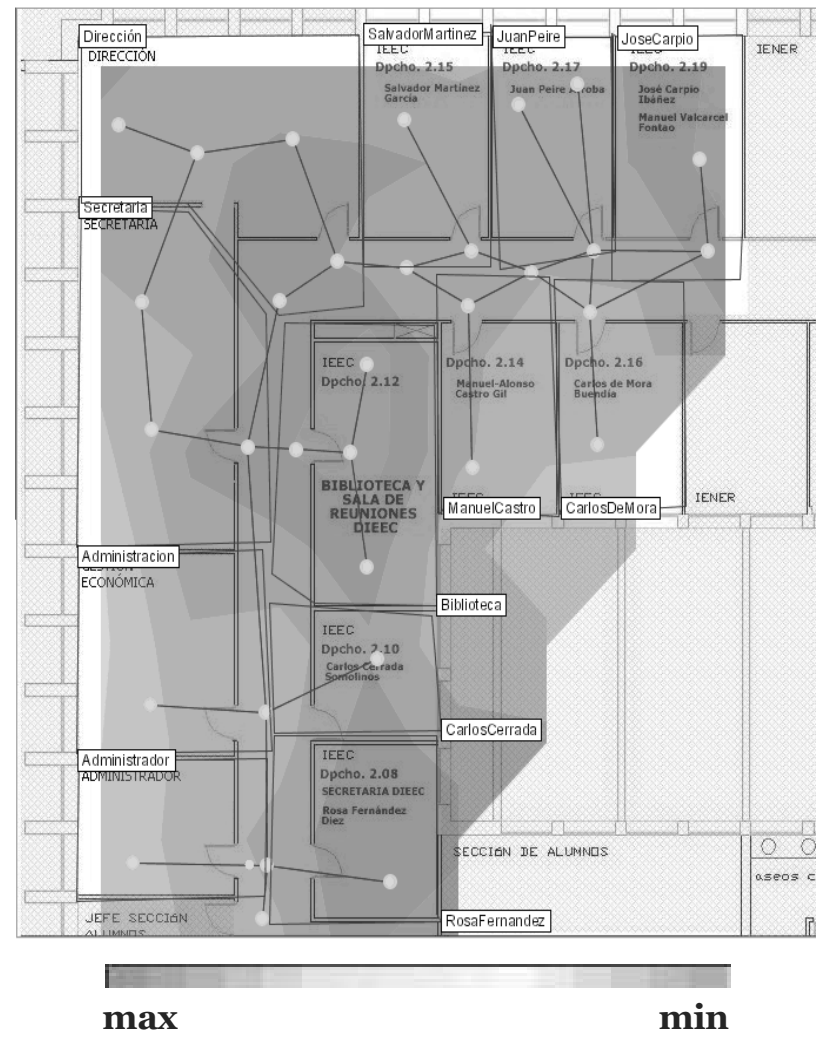

Figura 4: Mapa de cobertura con los puntos y caminos seguidos para realizar la calibración de la herramienta.

Una vez creado el patrón, la calibración se realiza tomando muestras de referencias del nivel de señal en cada punto (SRI) que son almacenadas en el módulo de posicionamiento (Ekahau Positioning Engine).

El Ekahau SDK proporciona en tiempo real las posiciones de cada dispositivo dentro del área de cobertura que son recogidas por el Gestor de Localización para su posterior filtrado (descartar posiciones 'no probables' o con un margen de error 
grande, etc.), tratamiento y envío al Gestor de Aplicaciones quién se encargará de, a partir de la posición del usuario, enviarle la información más adecuada adaptada, en formato y contenido, a las características de su terminal (una PDA, un ordenador portátil, un "Tablet PC", etc.).

Como ventajas de este sistema destacar su adaptación a condiciones cambiantes, su precisión (pueden conseguirse posicionamientos con errores menores de un metro) y las posibilidades que ofrece para definir áreas tridimensionales, es decir, sistemas que puedan cubrir varias plantas de uno o varios edificios.

Sin embargo, las pruebas realizadas también han mostrado ciertos inconvenientes como vibraciones de la posición ofrecida (por ejemplo, cuando el terminal se encuentra cerca de la frontera de dos o más zonas se detectan pequeños desplazamientos periódicos en la posición) o fluctuaciones esporádicas de importancia (de varios metros) de dicha posición cuando se alteran de manera significativa las condiciones (por ejemplo, cuando un gran número de personas entra simultáneamente en una sala vacía).

Para solucionar en la medida de lo posible estas desviaciones temporales (vibraciones y fluctuaciones), se han incorporado diversos algoritmos al sistema de gestión de la posición del software Ekahau que filtran posiciones "erróneas" como pequeños cambios periódicos en una posición o saltos "imposibles" en alguna de las coordenadas.

\section{Gestor de aplicaciones}

El último módulo necesario para el correcto funcionamiento de la herramienta es el gestor de aplicaciones, el cual provee las aplicaciones que se cargarán en la estación móvil del usuario y que, por tanto, le ofrecerán información personalizada en función de su localización geográfica (posición en el edificio o más en general en el campus). El Gestor de Aplicaciones se comunica por un lado con el navegador del terminal móvil y por otro con el sistema de localización. Esta información es ofrecida a través del navegador de Internet del terminal del usuario. Básicamente, la aplicación de la que dispondrá el usuario en su terminal se conectará al sistema de Gestión de Conocimiento de la universidad y seleccionará en él aquellas secciones relacionadas con su situación en el campus universitario, ofreciendo así la información de una forma personalizada en tiempo real. Por ejemplo, se ofrecerá:

- Información administrativa (matriculación, plazos y procedimientos) cuando se acceda a la secretaría del centro universitario. 
- Información sobre las ponencias cuando se acceda a cada una de las salas de conferencias.

- Prácticas que se realizan en cada uno de los laboratorios.

- Documentación sobre los cursos en cada una de las aulas.

- Elementos multimedia como videos y audio.

El Gestor de Aplicaciones ha sido desarrollado íntegramente en Java sobre tecnología XML. A él se podrá acceder desde cualquier navegador comercial de Internet. Esta tecnología proporciona la definición de una "capa" estándar por encima de aplicaciones dispares en diferentes entornos, para conseguir su interacción. Esta arquitectura abierta tiene, entre otras, las siguientes ventajas:

- Interoperabilidad: Esta tecnología está aceptada por todos los fabricantes, y por tanto permite operar entre aplicaciones de cualquier plataforma.

- Fácil integración entre los diferentes componentes y con aplicaciones externas.

- Escalabilidady portabilidad al estarbasada en estándares ampliamente aceptados.

- Accesibilidad desde cualquier navegador de Internet, por tanto no requiere instalaciones adicionales en los clientes (compatibilidad) y reduce el coste de implantación.

- También se reducen los costes al utilizar herramientas de libre difusión de código abierto como JAVA, Apache Tomcat Server y MySQL.

\section{EXPERIMENTACIÓN}

La carrera por dotar de mayores infraestructuras tecnológicas a las Universidades es un tema de gran importancia en la actualidad, es por ello que se decidió la implantación final del sistema en la primera planta del departamento de Ingeniería Eléctrica, Electrónica y de Control de la Escuela Técnica Superior de Ingenieros Industriales de la UNED, situado en la Ciudad Universitaria de Madrid (España) como parte de su plan de innovación tecnológica. Además de dicho departamento también se ha cubierto la zona de Dirección, Secretaría, Gestión Económica, Administración de la Escuela y Salón de Grados.

De esta manera se dotó de infraestructura inalámbrica posibilitando que tanto alumnos como profesores tuvieran acceso libre a Internet desde cualquier lugar de la planta, o incluso desde los jardines próximos al edificio. 


\section{Entorno Educativo}

El sistema se adaptó a las necesidades de la UNED, definiendo una serie de áreas lógicas escogidas estratégicamente dentro de la zona de despachos del departamento, de tal manera que cada despacho fuera un área lógica. El fin de tal distribución de áreas es proporcionar a los alumnos información relacionada con cada profesor o cada servicio ofrecido en la planta cubierta, por ejemplo, información de tutorías, teléfono, descripción de actividades realizadas, proyectos en los que trabaja, etc.

El siguiente paso dentro de la implantación del sistema en la UNED fue la creación de las aplicaciones asociadas a cada profesor o servicio, es decir, a cada área lógica. En este caso se optó por desarrollar también aplicaciones tipo web. En la figura 5 se muestran algunas de las aplicaciones web creadas.
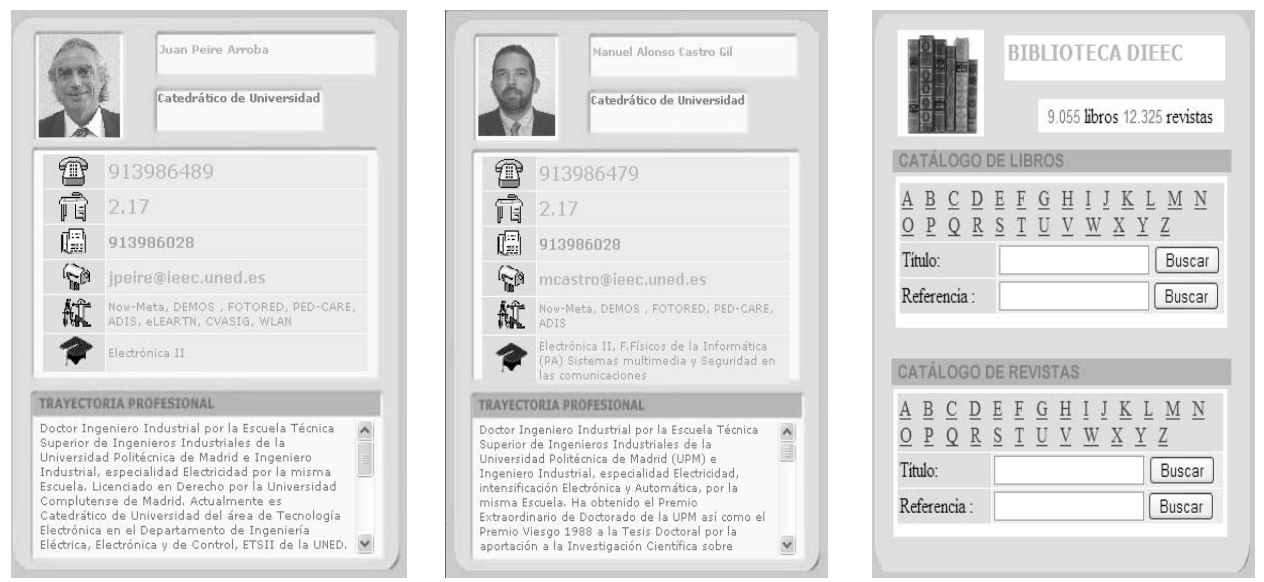

Fig. 5: Aplicaciones Web creadas para el entorno docente de la UNED

Además de estas fichas se crearon fichas interactivas para las áreas lógicas de dirección y jardines, ofreciendo audio y video en tiempo real al entrar en dichas áreas. Como valor añadido, además en el área lógica de Jardines se ofrece la posibilidad de acceder a Internet mediante un enlace a Google.

También se crearon aplicaciones de búsqueda dentro del catálogo de la biblioteca del departamento, permitiendo buscar por revistas o libros. En la imagen de la derecha de la figura 5 se puede ver la apariencia de dicha aplicación. 
Otra de las aplicaciones creadas fue una de tipo institucional, ofreciendo un video descriptivo de la UNED a la entrada del despacho del director de la escuela. Dicho video se ofrecía sin interacción ninguna por parte del usuario, y dependiendo del terminal que usara el usuario (portátil o PDA) se ofrecía un video de un tamaño o de otro. La aplicación desarrollada en la UNED ofrece diferentes posibilidades de configuración que pueden ser modificadas en cualquier momento.

En el caso de los navegadores Web, la opción más frecuente es la que combina un interfaz en el que se muestra la localización del usuario en un plano del edificio con otro en el que aparece la información asociada a la zona en la que se encuentra, (figura 6).

En caso de las agendas personales -PDAs- y dadas sus limitaciones gráficas, es más frecuente optar por una sola de estas interfaces, es decir, la que muestra el plano del edificio y la localización del usuario o la que ofrece la información asociada a la zona en la que se encuentra el usuario, tal y como se puede ver en la figura 7.

Una vez que el usuario se ha identificado se localiza al usuario dentro delárea cubierta y se le muestra la aplicación asociada a dicho mapa. En la siguiente figura se muestra el mapa con las distintas áreas definidas en la zona central, así como la localización del usuario dentro del mapa. Si el usuario no está dentro del área de cobertura en el lado derecho de la pantalla se mostrará un mensaje diciendo que se espere unos momentos, ya que no es posible mostrar la aplicación asociada al área, de lo contrario, aparecerá la aplicación asociada al área en el que se encuentre el usuario. En el lateral izquierdo superior se tiene el menú que permite salir de la aplicación, ver la ayuda o ir a la página de administración de la aplicación.

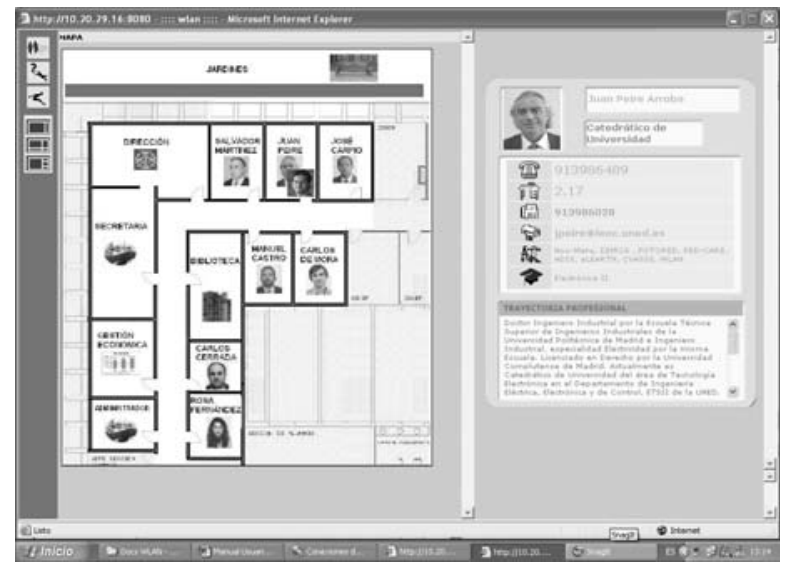

Figura 6: Pantalla de localización en el entorno docente de la UNED. 
Como se puede observar en la pantalla superior, el usuario se encuentra en el despacho de Juan Peire, con lo que en la parte derecha sale la aplicación asociada a dicha área, que en este caso es una página web con los datos de interés relacionados a este profesor. A medida que el usuario se vaya desplazando por el mapa definido, en el lado derecho de la pantalla aparecerá de manera dinámica la aplicación asociada a dicha área.

En la parte izquierda aparece el plano del edificio con las zonas disponibles (despachos, salas de reuniones y conferencias, laboratorios) así como la posición del usuario en tiempo real, en este caso situado frente a uno de los despachos. En la parte derecha aparece la información de contacto del profesor al que pertenece el despacho, información que se irá actualizando en tiempo real a medida que el usuario de desplace por el edificio.
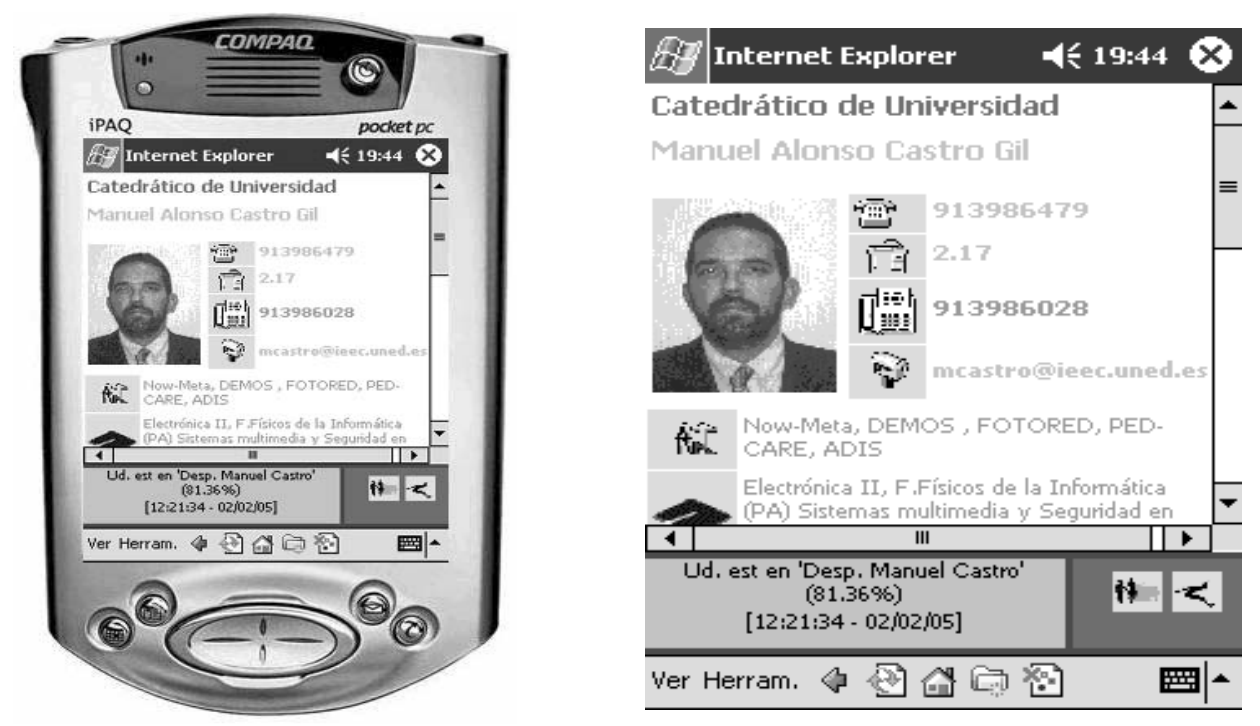

Fig. 7: Pantalla de PDA para un usuario situado en un despacho de un profesor.

Además de la localización el sistema posee un Menú General, (figura 8), desde el cual se tiene acceso a todos los servicios que ofrece la herramienta: Información General, Información de la Aplicación, Localización de Dispositivos y Gestión de la Aplicación, desde donde se puede obtener toda la información de cualquier usuario dentro del área de cobertura, realizar rastreos de caminos, gestión de actividades, así como la personalización total de la aplicación (cambiar colores, imágenes, idiomas, etc.). 


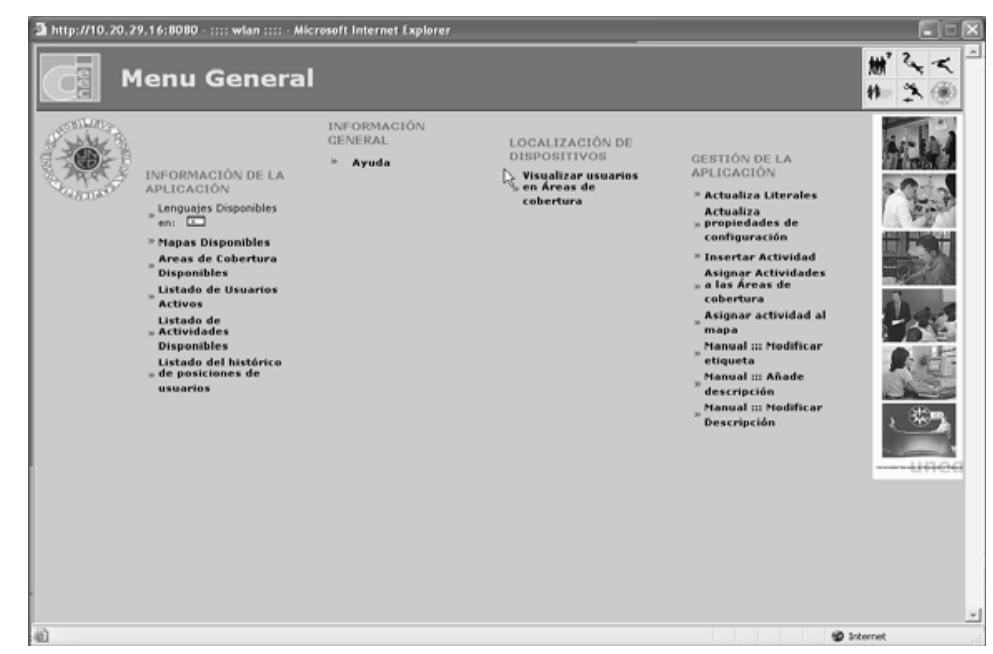

Fig. 8: Pantalla principal de gestión de la aplicación del entorno docente de la UNED.

En la actualidad, los alumnos están utilizando el sistema de localización de manera satisfactoria, ya sea mediante dispositivos móviles propios como teléfonos móviles, PDA's o ordenadores portátiles, o bien mediante equipos prestados por el departamento para su uso en las instalaciones. Además de la aplicación para los profesores se desarrollaron dos aplicaciones más como ejemplo del potencial de la herramienta: un museo y un hospital.

\section{Herramienta Entorno Museo}

El objetivo de la creación de una aplicación para un museo es que cuando un visitante entre en una sala sepa que obras de arte puede encontrar, así como una descripción de las mismas. La aplicación creada trata a cerca de un museo de Egipto, en el que se pueden encontrar distintas obras de arte relacionadas con el antiguo Egipto. Para su realización se definieron las áreas lógicas a usar en el mapa del museo, y una vez creadas las actividades asociadas a cada área lógica se enlazaron. A continuación mostramos una imagen del área de administración del sistema y otra de la pantalla que vería un usuario dentro del museo, (figuras 9 y 10). 


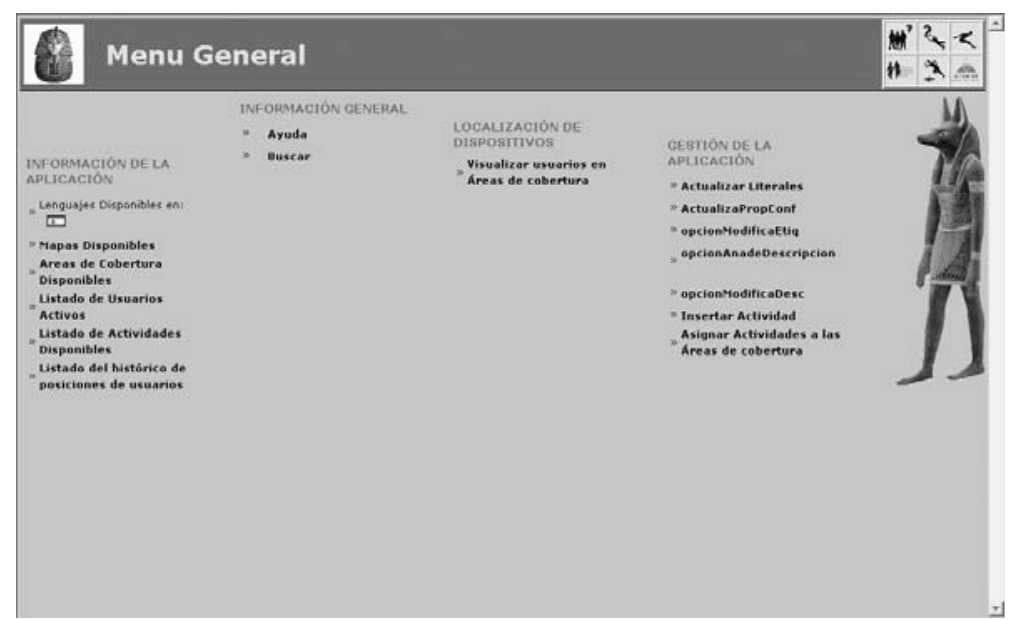

Fig. 9: Pantalla principal de administración del entorno Museo.

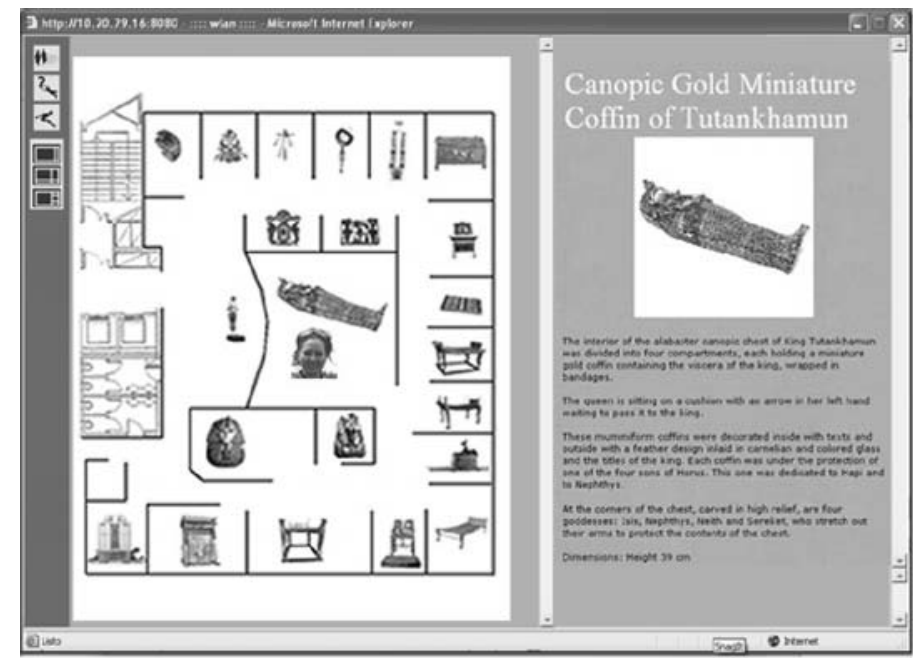

Fig. 1o. Pantalla de localización de usuarios del entorno Museo.

Como se puede ver en la figura, el visitante se encuentra en la sala del Sarcófago de Tutankhamon, con lo que el sistema al detectar su entrada muestra en la parte derecha de la pantalla la actividad asociada, que consiste en una página web con una explicación escrita al mismo tiempo que un fichero de audio en formato .mp3 lee la descripción. 
Esta aplicación fue implementada completamente, teniendo que realizar la adecuada personalización del sistema para adaptarse a los requerimientos que un museo tiene, la creación de las distintas actividades asociadas a las áreas, así como la instalación de la red inalámbrica correctamente securizada. A continuación se muestran algunas de las aplicaciones web que tuvieron que ser desarrolladas, (figura 11).
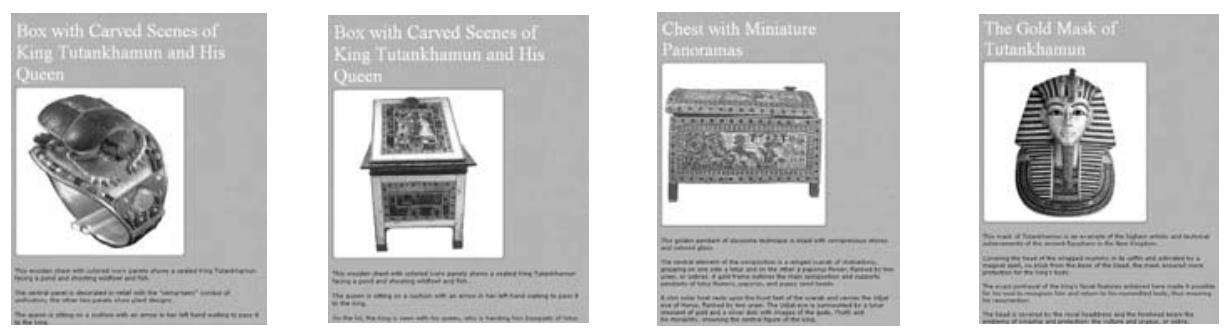

Fig. 11: Aplicaciones web creadas para el entorno Museo.

\section{Herramienta Entorno Sanitario}

En este entorno se pretendía realizar un seguimiento de pacientes que llevan sistemas de alarma para emergencias, del personal de hospitales que llevan agendas electrónicas, así como de los dispositivos telemétricos en una red WLAN. En la figura 12 se puede observar una captura de pantalla de dicha aplicación y en al figura 13 el Menú General de la aplicación.

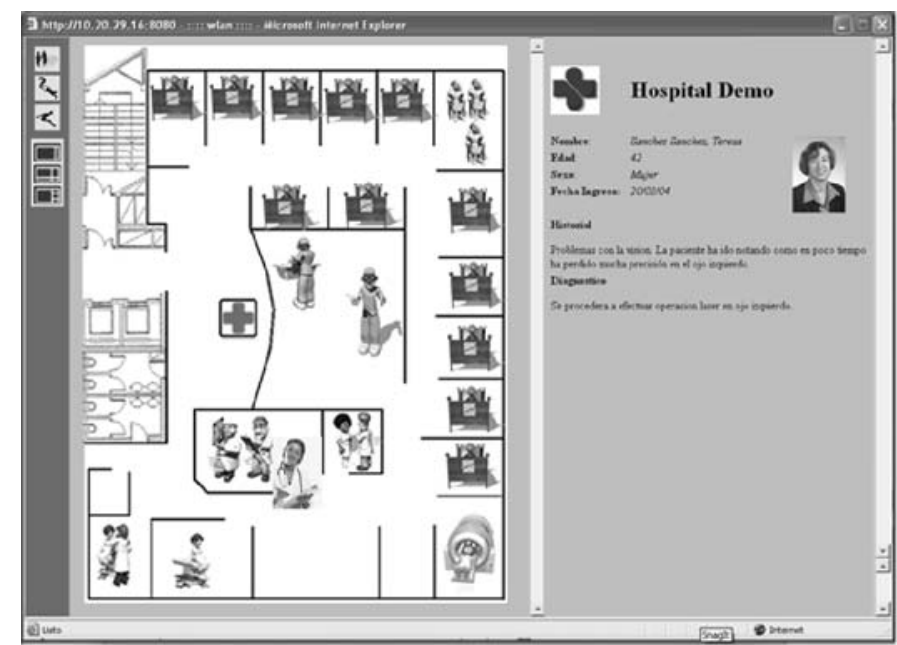

Fig. 12: Pantalla de localización de usuarios del entorno sanitario. 


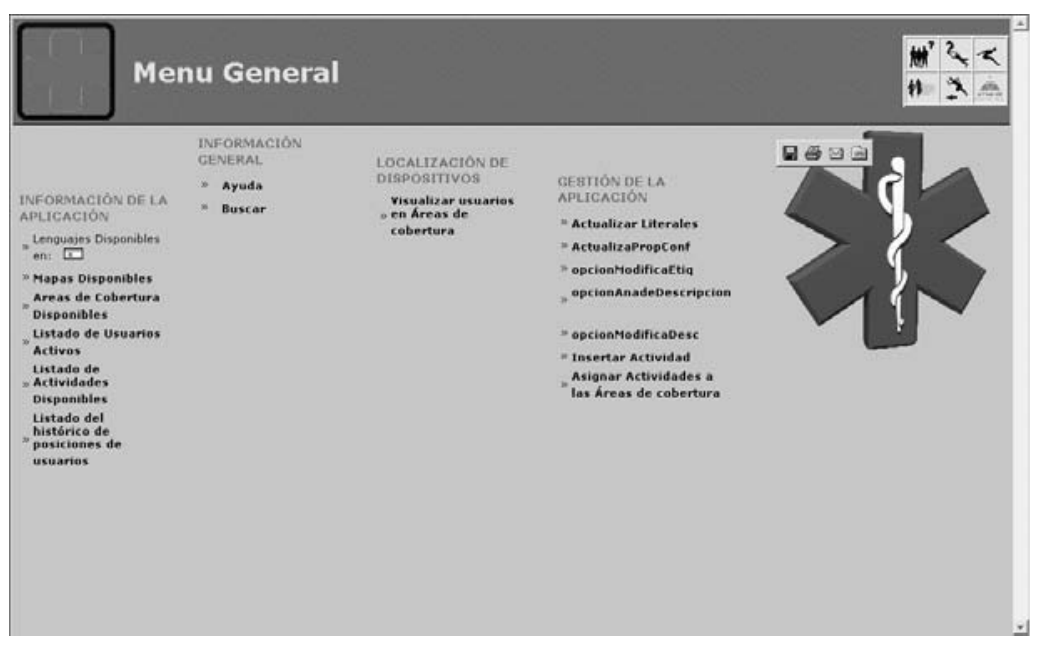

Fig. 13: Pantalla principal de administración del entorno Sanitario.

$\mathrm{Al}$ igual que en el caso del museo, esta aplicación fue implementada a partir de la creación de un mapa de áreas lógicas adecuadamente definidas, junto con el desarrollo de las fichas de pacientes hospitalizados, mostradas a continuación en la figura 14.
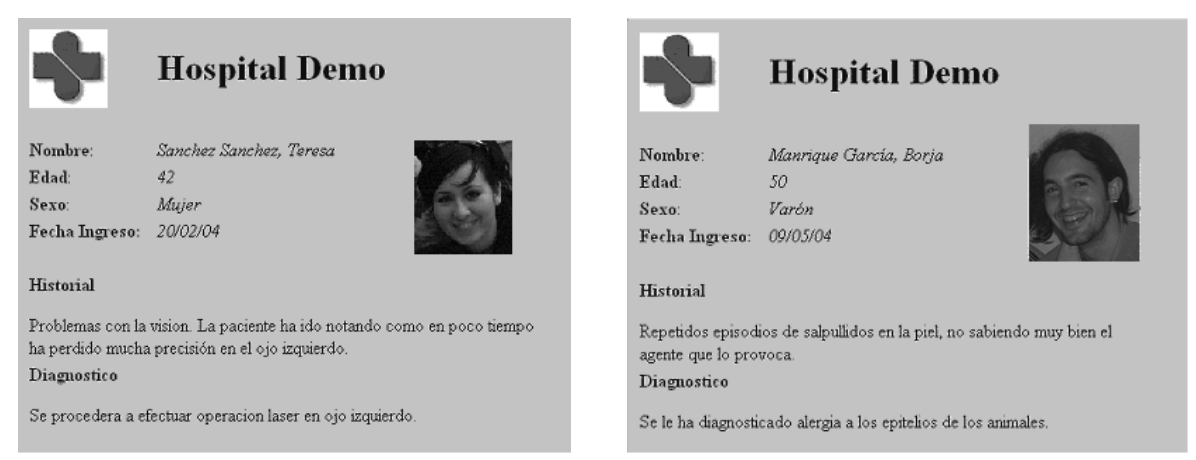

Fig. 14: Aplicaciones web creadas para el entorno Sanitario.

\section{NOVEDAD TECNOLÓGICA}

Las posibles aplicaciones derivadas de la localización de usuarios dentro del área global de cobertura de una red están siendo ampliamente estudiadas al ofrecer 
un gran número de ventajas en cuanto a la mejora del rendimiento de la red y a la comodidad que supone, para los usuarios, el acceso a información actualizada e incluso personalizada en función de sus coordenadas geográficas. Entre los servicios basados en localización geográfica que podrían ofrecerse podemos citar los servicios de seguridad (emergencia, asistencia en carretera, vigilancia forestal, etc.), servicios de búsqueda (vehículos, personas), encaminamiento de llamadas al centro más cercano (envíos de comida, servicios de asistencia técnica, etc.) o servicios de información personalizada (páginas amarillas, información turística, publicidad localizada, etc.).

Sin embargo, el terreno del desarrollo de servicios basados en localización con tecnología de red inalámbrica WLAN está aún sin explorar. Existen herramientas comerciales que proporcionan las coordenadas de un individuo equipado con una tarjeta inalámbrica dentro de un recinto cubierto por varios puntos de acceso. No obstante, no existen aplicaciones directamente relacionadas con el uso de estas coordenadas para la gestión de la red, o para la obtención de información personalizada, entre otras.

El empleo de un Gestor de Aplicaciones en función de la localización del usuario aplicado a campus universitarios resulta también muy novedoso. De esta forma, un usuario que, por ejemplo, se encuentre en la sala de conferencias de la universidad, tendrá acceso directo a la información relativa al contenido del evento. Por otro lado, ese mismo usuario al entrar en un laboratorio, tendrá acceso directo a los contenidos de la práctica que se esté llevando a cabo. En consecuencia, se ofrece a los usuarios la flexibilidad de tener distintos perfiles y permisos según donde estén ubicados. Al tener definidas zonas para usuarios y servicios concretos se asegura la integridad del sistema, dado que el acceso al mismo estará controlado en cada momento.

Por último, destacar que el potencial de este tipo de arquitecturas basadas en localización es enorme. Al margen de las aplicaciones de gestión de servicios desarrolladas en el entorno universitario de la UNED, se abren nuevas posibilidades como las que aquí se citan, algunas de las cuales ya se han mencionado:

- Hospitales: por ejemplo, un médico o enfermero podría moverse por distintas salas o habitaciones con su PDA, y acceder directamente a las historias clínicas de los pacientes que deben ser atendidos en la sala o que estén ingresados en la habitación a la que acaba de entrar.

- Museos: por ejemplo, un visitante equipado con su portátil o su PDA que se encuentre en la sala $62^{\text {a }}$ del museo del Prado, podría obtener la información de "La Trinidad" y de su autor, el Greco, automáticamente por el hecho de encontrarse en dicha sala. 
- Instituciones públicas: por ejemplo, los políticos en el Senado podrían acceder directamente a un listado de enlaces y a documentos relacionados con la nueva ley que se esté debatiendo, etc.

- Industria: se podría acceder directamente a las especificaciones y manuales de uso de la maquinaria de una instalación, etc.

- Recintos feriales: se facilitaría la información detallada del pabellón o stand que se esté visitando, el envío de publicidad e incluso noticias en tiempo real, por ejemplo, el anuncio de una charla en determinado pabellón, etc.

Logística: se optimizarían las labores de seguimiento de inventariado de stocks, etc.

\section{CONCLUSIONES}

El desarrollo de proyectos relacionados con estas tecnologías mantienen a la UNED en la vanguardia tecnológica, sirviendo de punta de flecha para la llegada de estos sistemas a otras áreas de la sociedad, como pueden ser el ámbito sanitario, museos, vigilancia de edificios, información turística en las ciudades, centros de congresos, centros de atención al cliente, y todos aquellos ámbitos en los que se precisa una personalización de la atención al usuario.

No obstante, para que los gestores de aplicaciones basados en localización mediante redes inalámbricas lleguen a sectores comerciales parece necesaria una mejora en la precisión de la localización, así como una mayor robustez a la hora de soportar perturbaciones externas. Algunas de las tecnologías que se podría emplear como sustitutivo de las redes wi-fi 802.11b podrían ser redes bluetooth, infrarrojos, redes inalámbricas WAVELAN DE AT\&T que actúan en la banda de 902-928 Mhz o redes HyperLan que pueden actuar en la frecuencia de banda de $5 \mathrm{GHz}$. De esta manera, al cambiar la banda de frecuencia en la que se trabaja se podría evitar que la red sufriera variaciones de potencia en función de los entornos ambientales, tales como el número de personas alrededor. 


\section{REFERENCIAS BIBLIOGRÁFICAS}

Departamento de Ingeniería Eléctrica, Electrónica y de Control [en línea]. Disponible en: http://www.ieec.uned. es/ [consulta 2006, 03 de Mayo]

Ekahau Positioning Engine [en línea]. Disponible en: http://www.ekahau.com/ ? id=4500. [consulta 2006, o3 de Mayo]

Gimeno, J. M. (2004). Computación Ubicua [en línea]. Disponible en: http://www. laflecha.net/canales/ciencia/articulos/ computacion ubicua [consulta 2006, 03 de Mayo]

Martín, S.; Castro, M.; Peire, J. y otros (2005). Experiencias e introducción de dispositivos móviles en la Enseñanza a Distancia. Simposio sobre Computación Ubicua e Inteligencia Artificial Ambiental UCAmI'o5. Granada, España.
Martín, S., Castro, M. y otros (2005). Development and implementation of an application manager using location by wireless LAN. eChallenges 2005, Ljubljana Slovenia.

Rivilla, I., Castro, M. y otros (2004). Development and implementation of a collaborative environment for education.eAdoption and the Knowledge Economy - Issues, Applications, Case Studies. Editores: Cunningham, P y Cunnningham, M. Ed. IOS Press, Amsterdam Holanda.

Universidad Nacional de Educación a Distancia [en línea]. Disponible en: http://www.uned.es/ [consulta 2006, o3 de Mayo]

\section{PALABRAS CLAVE}

Redes inalámbricas, Localización geográfica, UNED, Plataforma abierta, Servicios, Alumnos, Profesores.

\section{KEYWORDS}

Wireless lan, Geographic location, Open platform, Services, Students, Teachers.

\section{PERFIL ACADÉMICO DE LOS AUTORES}

\section{Sergio Martín Gutiérrez}

Ingeniero Superior en Informática por la Universidad Carlos III de Madrid. Ha participado desde 2002 en proyectos de investigación nacionales e internacionales en la UNED. Dichos proyectos estaban relacionados con redes inalámbricas, "e-learning" y nuevas tecnologías. Así mismo, ha publicado artículos en congresos tanto nacionales como internacionales sobre dichos temas. En la actualidad está cursando estudios de 
Doctorado en el Departamento de Ingeniería Eléctrica, Electrónica y de Control de la UNED.

\section{Manuel A. Castro Gil}

Doctor Ingeniero Industrial y Catedrático de Universidad. Ha sido Vicerrector de Nuevas Tecnologías de la UNED, así como Subdirector de Ordenación Académica y de Investigación, y Director del Centro de Servicios Informáticos de la UNED. Ha participado en numerosos proyectos de investigación como investigador, coordinador y director y ha publicado en revistas y congresos, tanto nacionales e internacionales. Ha publicado igualmente diversos libros y material multimedia dentro de sus líneas de investigación y docencia. Es Senior Member del IEEE.

\section{Rosario Gil Ortego}

Ingeniero de Telecomunicación por la Universidad de Alcalá de Henares (Madrid). Obtuvo Matrícula de Honor con el proyecto "Comportamiento de los códigos LDPC sobre canales Rayleigh". Ha sido consultor junior en Axpe Consulting. En la actualidad está cursando estudios de Doctorado y participa en proyectos de investigación relacionados con la biometría aplicada a la tele-educación en el Departamento de Ingeniería Eléctrica, Electrónica y de Control de la UNED.

\section{Juan Peire Arroba}

Doctor Ingeniero Industrial, Licenciado en Derecho y Catedrático de Universidad del área de Tecnología Electrónica en la UNED. Ha recibido el premio a la "Innovative Excellence in Teaching, Learning \& Technology" del "Center for the Advancement of Teaching and Learning” del año 1999. Ha trabajado varios años como Consultor especializado en la creación de Empresas Tecnológicas, así como ha dirigido y dirige diversos proyectos de investigación relacionados con e-learning y nuevas tecnologías, tanto nacionales como internacionales.

Dirección postal: $\quad$ Departamento de Ingeniería Eléctrica Electrónica y de Control Escuela Técnica Superior de Ingenieros Industriales Universidad Nacional de Educación a Distancia C/ Juan del Rosal, 12, Ciudad Universitaria 28040 Madrid. España

E-mail: $\quad$ smartin@ieec.uned.es mcastro@ieec.uned.es

rgil@ieec.uned.es jpeire@ieec.uned.es

Fecha recepción del artículo: 23. 10. 06

Fecha aceptación del artículo: 03.12. 06 